\title{
FLUJOS DE C, N Y P AL SUELO DE DOS ESPECIES DE ÁRBOLES REMANENTES EN UNA PRADERA TROPICAL ESTACIONAL
}

\author{
LeOpoldo Galicia \\ Instituto de Geografía \\ Universidad Nacional Autónoma de México \\ Apartado postal 20-850 \\ 04510 México, D.F. \\ Felipe García-Oliva ${ }^{1}$ \\ Instituto de Ecología \\ Universidad Nacional Autónoma de México \\ Apartado postal 27-3 \\ Santa María de Guido \\ 58090 Morelia, Michoacán, México \\ e-mail: fgarcia@ate.oikos.unam.mx \\ RAMÓN MURILLO \\ Y \\ Magdalena Oliva \\ Departamento de Biología \\ Facultad de Química \\ Universidad Nacional Autónoma de México \\ 04510 México, D.F.
}

\section{RESUMEN}

Se analizaron las concentraciones de C, N y $\mathrm{P}$ total de hojas vivas, hojarasca y mantillo de dos especies de árboles en individuos remanentes en medio de una pradera en un ecosistema tropical estacional en la costa de Jalisco, México. Las especies tuvieron diferencias en la dinámica de nutrientes: Cordia elaeagnoides presentó una mayor concentración de $\mathrm{P}$ en las hojas vivas, hojarasca y mantillo que Caesalpinia eriostachys, pero esta última registró una mayor eficiencia de uso de P. En contraste, el flujo de nutrientes por medio de la hojarasca varió entre años, sugiriendo que la productividad de un periodo en particular determina la cantidad de nutrientes que retornan al suelo. Sin embargo, la magnitud de este efecto puede quedar reducida por el corto tiempo de residencia de la materia orgánica y de los nutrientes del mantillo asociado a ambas especies de árboles.

\footnotetext{
1 Autor encargado.
} 
Acta Botanica Mexicana (2002), 61: 41-57 nutrientes.

Palabras clave: trópico estacional, hojarasca, mantillo, materia orgánica, eficiencia de uso de

\begin{abstract}
We investigated the differences between two trees species in total $\mathrm{C}, \mathrm{N}$ and $\mathrm{P}$ concentrations in live leaves, literfall, and surface litter. The work was conducted on individuals remnant in pastures, in a tropical deciduous forest ecosystem on the coast of Jalisco, Mexico. The use of nutrients differed between the two species: Cordia elaeagnoides had higher $\mathrm{P}$ concentration in live leaves, litterfall and surface litter than Caesalpinia eriostachys, but the latter species had a higher $\mathrm{P}$ use-efficiency. In contrast, the amount of nutrients fluxed through litterfall varied among years, suggesting that the productivity of specific year determined the amount of nutrients returned to the soil. However, the magnitude of this effect can be depleted by the short residence time of organic matter and nutrients of surface litter associated to both species.
\end{abstract}

Key words: Seasonal tropic, litterfall, litter, organic matter, nutrient use-efficiency.

\title{
INTRODUCCIÓN
}

Existe una fuerte relación entre la forma como las especies de plantas usan los nutrientes y su ciclaje en los ecosistemas terrestres (Stone, 1975; Vitousek, 1982,1984; Pastor et al., 1984; Shaver y Melillo, 1984; Miles, 1985; Binkley y Valentine, 1991). Por ejemplo, se ha propuesto una retroalimentación positiva entre el flujo de los nutrientes de la planta y su disponibilidad en el suelo (Hobbie, 1992). Sin embargo, el efecto de cada especie vegetal en la dinámica de nutrientes depende de las características del suelo y del nutriente que se trate (Binkley et al., 1992; Koch y Matzner, 1993; GarcíaMontiel y Binkley, 1998; Døckersmith et al., 1999). La influencia de diferentes tipos de plantas sobre tales procesos edáficos ha sido examinada en ecosistemas áridos (GarcíaMoya y McKell, 1970; West, 1981; Burke et al., 1989), en sabanas tropicales (Kellman, 1979; Belsky, 1994; García-Miragaya et al., 1994) y en selvas bajas caducifolias (Døckersmith et al., 1999), en los que se ha demostrado la fuerte influencia de las especies vegetales en la dinámica de los nutrientes del suelo. Entre los mecanismos más importantes que utilizan las plantas en la economía de los nutrientes está la re-utilización de una fracción de los existentes en sus hojas vivas para generar más biomasa. La producción de biomasa por unidad de nutriente absorbido se define como eficiencia de uso de nutrientes y es reconocida como uno de los principales mecanismos de protección de nutrientes que tienen las plantas (Vitousek, 1982). La eficiencia de uso de nutrientes afecta la composición química de las hojas, y así cuando dominan las moléculas más recalcitrantes, disminuye la tasa de liberación de nutrientes al suelo (Hobbie, 1992). Por ejemplo, se puede incrementar la concentración de lignina, cuya molécula es difícil de descomponer por las poblaciones microbianas del suelo (Stevenson, 1994), por lo que esta substancia ha sido utilizada como índice de la calidad del material vegetal (Melillo et al., 1982; Parton et al, 1987). Asimismo se ha reconocido a los polifenoles como compuestos que pueden limitar la actividad microbiana, principalmente en especies tropicales de la familia Leguminosae (Palm y Sánchez, 1990). 
Durante la década pasada, se han desarrollado varios trabajos que reconocen el papel de los árboles remanentes como núcleos para la restauración de los ecosistemas, debido a que mantienen una serie de procesos ecológicos del bosque debajo de sus copas (McDonell, 1986; Campbell et al., 1990, Guevara et al., 1992; Otero-Arnaíz et al., 1999). Sin embargo, poco se ha estudiado cómo las especies de árboles remanentes afectan el ciclaje de nutrientes y su papel potencial en la restauración funcional de los ecosistemas tropicales. Para responder estas preguntas es necesario describir primero la forma cómo las especies individuales de árboles remanentes utilizan los nutrientes. El objetivo del presente trabajo es comparar las concentraciones totales de $\mathrm{C}, \mathrm{N}$ y $\mathrm{P}$ foliar y su flujo al suelo a través de la caída de hojarasca de dos especies de árboles en una pradera, que representan individuos remanentes de la selva natural en un ecosistema tropical estacional.

\section{MÉTODOS}

Sitio de estudio

El material vegetal fue colectado en el ejido de San Mateo, municipio La Huerta en el estado de Jalisco $\left(19^{\circ} 29^{\prime} \mathrm{N}\right.$ y $\left.10^{\circ} 0^{\prime} \mathrm{O}\right)$. El relieve se caracteriza por presentar lomeríos con pendientes convexas (Rodríguez, 1999). El tipo de suelo dominante es entisol (USDA; Solís, 1993), sobre rocas riolíticas del Terciario (Campo et al., 2001). La temperatura media anual del aire es de $25^{\circ} \mathrm{C}$ (Bullock, 1986). La precipitación promedio anual es de $767 \mathrm{~mm}$ (1983-2000), concentrándose $80 \%$ de las lluvias entre junio y octubre (García-Oliva et al., 1995a; Fig. 1), de las que una parte obedece a la influencia de los ciclones tropicales del Pacífico (García-Oliva et al., 1991). El tipo principal de vegetación es selva baja caducifolia (Miranda y Hernández X., 1963), la cual pierde la mayoría de sus hojas cuando termina la estación de lluvias, entre los meses de noviembre y diciembre (Bullock y Solís-Magallanes, 1990; Martínez-Yrízar y Sarukhán, 1990) y cuya productividad primaria neta es de $12,060 \mathrm{~kg} \mathrm{ha}^{-1} \mathrm{a}^{-1}$, de la cual $43 \%$ es subterránea (Martínez-Yrízar et al., 1996).

La selva se transforma en praderas por medio del proceso de la roza-tumbaquema seguido por la siembra de gramíneas exóticas. Las especies de pastos más utilizadas son guinea (Panicum maximum Jacq.) y buffel (Cenchrus ciliaris L.) para uso ganadero (Gutiérrez-Alcalá, 1993). Una vez establecida la pradera, se le quema aproximadamente cada dos años para evitar la invasión de malezas, dependiendo de qué tan húmedo fue el año. Durante la transformación de la selva en pradera se dejan árboles aislados en pie, los cuales se utilizan para sombra de ganado. El suelo debajo de las copas de los árboles tiene mayor disponibilidad de nutrientes que el ubicado fuera de sus copas (Galicia, 2001); sin embargo, el pH y las concentraciones de las formas totales de C, N y P del suelo bajo las copas de los árboles fueron similares (Cuadro1, Galicia, 2001). El suelo sufre cambios importantes durante la quema (García-Oliva et al., 1999a y 1999b, Giardina et al. 2000) y continúa su degradación durante su uso continuo, principalmente en términos de la disminución de la materia orgánica (García-Oliva et al., 1994) y de los contenidos de nutrientes (García-Oliva y Maass, 1998), siendo la erosión hídrica uno de los principales procesos de degradación (Maass et al., 1988; García-Oliva et al., 1995b). 


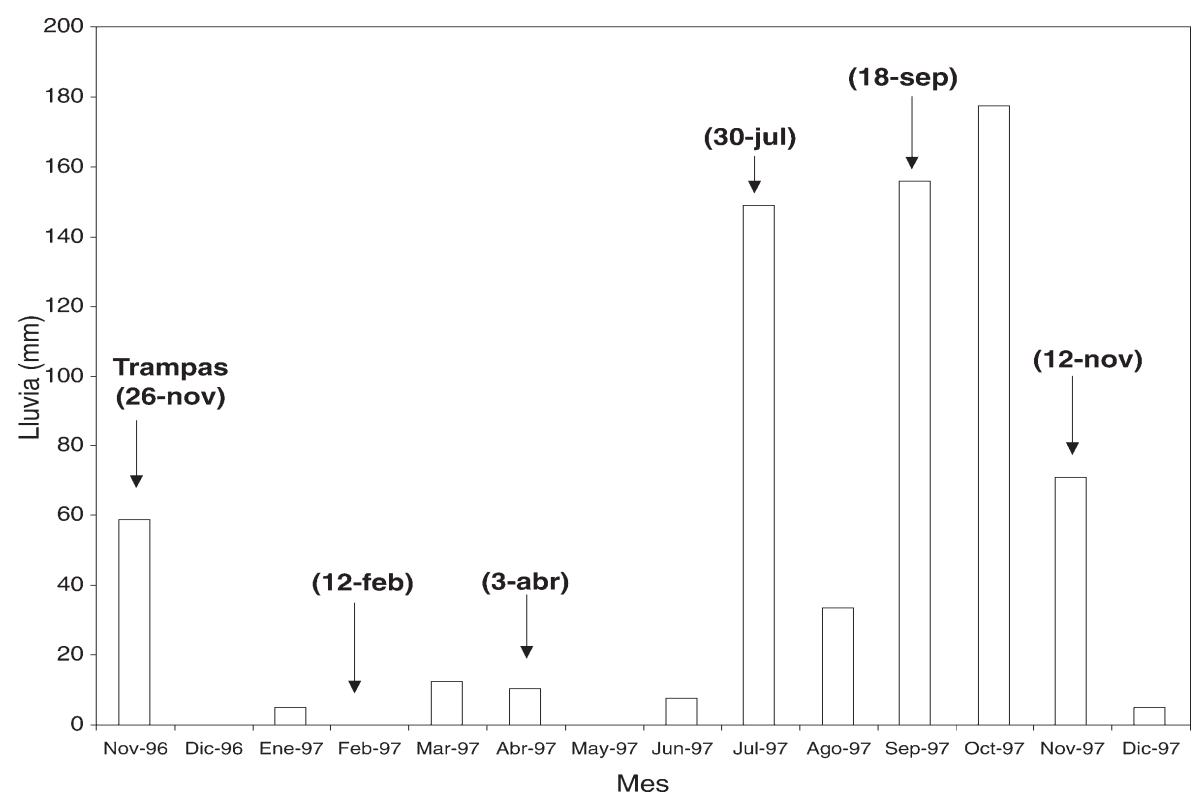

Fig. 1. Cantidad de lluvia mensual $(\mathrm{mm})$ durante el periodo de estudio en la región de Chamela, Jalisco (nov-96 a nov-97). Las fechas corresponden a la colocación de las trampas de hojarasca (26 de noviembre de 1996) y a los cinco muestreos.

\section{Muestreo}

Las especies de árboles utilizadas en este estudio fueron Caesalpinia eriostachys Benth. (Leguminosae) y Cordia elaeagnoides DC. (Boraginaceae), las cuales fueron identificadas de acuerdo con el listado florístico de Lott (1993) de la región de Chamela. Se les escogió por su importancia en la estructura y productividad de la selva (MartínezYrízar, 1984) y porque son dos de las cuatro plantas más comunes que se acostumbra dejar como árboles remanentes en las praderas de esta región (González-Flores, 1992). Asimismo, estas especies tienen características foliares contrastantes, que pueden afectar su tasa de descomposición y por lo tanto el reingreso de nutrientes al suelo (MartínezYrízar, 1984). Caesalpinia eriostachys es un árbol caducifolio y con hojas compuestas bipinnadas; mientras que Cordia elaeagnoides tiene hojas anchas simples igualmente caducas en la temporada seca (Martínez-Yrízar, 1984; Lott, 1993). Se localizaron cinco parcelas con más de siete años de manejo continuo y de aproximadamente 5 hectáreas de superficie, en que ambas especies estuvieran presentes y que la distancia mínima entre árboles fuera de 20 metros. Se eligió un individuo maduro de cada especie por parcela. Los árboles de $C$. eriostachys tuvieron una altura de 6 a $8 \mathrm{~m}$ y un DAP (diámetro a la altura del pecho) entre 0.11 y $0.43 \mathrm{~m}$. Los árboles de $C$. elaeagnoides midieron 8 a $10 \mathrm{~m}$ de alto y su DAP fue de 0.86 a $0.95 \mathrm{~m}$. Se consideró a la parcela como la unidad experimental. Los individuos seleccionados se excluyeron del ganado con un cerco de 
Galicia et al.: Flujos al Suelo de Dos Especies de Árboles en una Pradera Tropical Estacional

alambre de púas dejando una superficie interior de $2.25 \mathrm{~m}^{2}$, para evitar que se destruyeran las trampas de muestreo. De cada árbol se recogieron hojas vivas, hojarasca y mantillo en diferentes fechas. Las hojas vivas se colectaron manualmente a variadas alturas de la copa y en áreas de diferente exposición a la radiación solar de todos los individuos en los meses de julio y septiembre de 1997. Debajo de cada individuo se ubicó una trampa de hojarasca de $50 \mathrm{~cm}$ de diámetro, con $50 \mathrm{~cm}$ de fondo y a un metro de altura del piso, fabricada de nylon, siguiendo el diseño IBP (Newbould, 1967), obteniéndose un área de colecta de $0.196 \mathrm{~m}^{2}$. Las trampas fueron colocadas en noviembre de 1996 y se vaciaron en cinco ocasiones: febrero, abril, julio, septiembre y noviembre de 1997. El mantillo se recogió del suelo utilizando un plato de $20 \mathrm{~cm}$ de diámetro para definir un área de colecta de $0.031 \mathrm{~m}^{2}$, misma que se realizó en los meses de abril, julio, septiembre y noviembre de 1997. En cada una de las fechas se obtuvo la muestra en un sitio debajo de la copa de cada árbol, que se asignó al azar, evitando que estos lugares fueran afectados por la trampa de hojarasca y las colectas de fechas anteriores. El material de las hojas vivas, la hojarasca y el mantillo se secaron, pesaron y molieron por separado para su análisis químico. Las muestras de hojarasca y mantillo fueron revisadas manualmente para eliminar hojas que no pertenecieran a cada una de las especies estudiadas, procedimiento que fue particularmente fácil debido al aislamiento de los árboles dentro de la pradera. Los análisis químicos de la hojarasca de febrero y abril y del mantillo de abril se realizaron con una muestra compuesta de los cinco árboles de la misma especie, que se molió conjuntamente para obtener una mezcla homogénea.

Cuadro 1. Media \pm un error estándar de las características químicas del suelo asociado a las dos especies de árboles remanentes en condiciones de pradera en Chamela, Jalisco (Galicia, 2001). No existieron diferencias significativas entre especies $(p=0.05, n=5)$.

\begin{tabular}{|l|l|l|}
\hline & \multicolumn{1}{|c|}{ C. elaeagnoides } & \multicolumn{1}{c|}{ C. eriostachys } \\
\hline $\mathrm{pH}\left(\mathrm{H}_{2} \mathrm{O}\right)$ & $7.45 \pm 0.15$ & $7.13 \pm 0.24$ \\
$\mathrm{C}\left(\mu \mathrm{g} \mathrm{g} \mathrm{g}^{-1}\right)$ & $31,061 \pm 2,598$ & $29,854 \pm 2,996$ \\
$\mathrm{~N}\left(\mu \mathrm{g} \mathrm{g}^{-1}\right)$ & $2,179 \pm 44$ & $2,086 \pm 314$ \\
$\mathrm{P}\left(\mu \mathrm{g} \mathrm{P}^{-1}\right)$ & $520 \pm 62$ & $383 \pm 34$ \\
$\mathrm{C}: \mathrm{N}$ & 14.2 & 14.3 \\
$\mathrm{C}: \mathrm{P}$ & 59.7 & 77.9 \\
$\mathrm{~N}: \mathrm{P}$ & 4.9 & 5.4 \\
\hline
\end{tabular}

Análisis de laboratorio

Se hicieron las siguientes determinaciones químicas cuantitativas: carbono total (C) con un analizador automatizado de $\mathrm{CO}_{2}$ UIC Mod. CM5012; nitrógeno (N) y fósforo (P) total con el método semi-Kjeldahl modificado para autoanalizadores (Anónimo, 1977), por medio de digestión ácida y leídos por colorimetría; lignina y celulosa por medio del método de detergente de fibra ácida (Anderson e Ingram, 1993) y polifenoles con el método Folin-Denis (Anderson e Ingram, 1993). 


\section{Análisis estadísticos}

Se aplicó un análisis de varianza factorial (ANOVA) para determinar las diferencias entre especies de árboles y fechas, por lo que los factores fueron especies de árboles remanentes (con dos niveles) y fechas. En el caso de este último factor, los niveles cambiaron de acuerdo con las variables: en hojas vivas fueron dos (junio y septiembre), en la hojarasca fueron cinco (febrero, abril, junio, septiembre y noviembre) y en el caso del mantillo fueron cuatro (abril, junio, septiembre y noviembre). Las comparaciones entre las medias se realizaron con la prueba de Tukey (Montgomery, 1984). La eficiencia de uso de nutrientes fue calculada como la masa de la hojarasca entre el contenido del nutriente en la hojarasca expresado en masa (Vitousek, 1982). Se estimó el tiempo de residencia de la materia orgánica y del contenido de nutrientes con la siguiente fórmula (Vogt et al., 1986): TR= $(\mathrm{M} / \mathrm{H})$, donde $\mathrm{M}$ es igual al promedio del contenido de mantillo de junio a noviembre de 1997 y $\mathrm{H}$ es la cantidad de producción de hojarasca entre junio y noviembre de 1997. Para el caso del tiempo de residencia de los nutrientes, se utilizaron los valores de contenido de nutrientes respectivos en el mantillo y la hojarasca.

\section{RESULTADOS}

Hojas vivas

Los nutrientes analizados en las hojas vivas de los meses de julio y septiembre para ambas especies muestran que $C$. eriostachys presentó mayor concentración de C que $C$. elaeagnoides $(p=0.0001)$, mientras que el patrón fue inverso para el $P$ en ambas fechas $(p=0.003$, Cuadro 2). En cambio, las diferencias entre las especies en la concentración de $\mathrm{N}$ no fueron significativas $(p=0.27)$. C elaeagnoides tuvo menores cocientes C: $P$ y N: $P(p=0.0001$ y $p=0.01$, respectivamente).

Las diferencias de concentración de celulosa entre las especies no fueron significativas $(p=0.08)$. En contraste, las hojas de $C$. elaeagnoides tuvieron mayor cantidad de lignina que $C$. eriostachys $(p=0.001)$. Por su parte, la concentración de los polifenoles presentó un patrón inverso entre las especies ( $p=0.0001$; Cuadro 2). Como consecuencia, los cocientes lignina: celulosa y lignina: nitrógeno fueron mayores en $C$. elaeagnoides que en $C$. eriostachys en ambas fechas ( $p=0.007$ y $p=0.009$, respectivamente). Curiosamente, la concentración de lignina no varió entre las fechas de colecta $(p=0.93)$ a diferencia de la celulosa, que fue mayor en las hojas de septiembre ( $p=0.01$; Cuadro 2). En cambio, el término de la interacción (especie por fecha) fue significativo en el caso de los polifenoles $(p=0.0001)$, reduciéndose cerca de $60 \%$ en C. elaeagnoides y aumentando casi $10 \%$ en $C$. eriostachys en las muestras de septiembre (Cuadro 2).

\section{Dinámica de la hojarasca}

La concentración de $\mathrm{C}$ y $\mathrm{P}$ en la hojarasca de cada especie presentó el mismo patrón que en las hojas vivas (Cuadro 3): C. eriostachys tuvo una mayor concentración 
de $C$ que $C$. elaeagnoides en todas las fechas $(p=0.003)$ y lo opuesto se encontró para la concentración de $\mathrm{P}(\mathrm{p}=0.01)$. En cambio, el patrón del $\mathrm{N}$ no fue muy claro, ya que en las muestras de febrero y abril, $C$. elaeagnoides tuvo la mayor concentración y en las muestras de septiembre y noviembre la ventaja correspondió a $C$. eriostachys $(p=0.04)$. Por lo anterior, el término de la interacción del cociente $C: N$ fue significativo $(p=0.001)$ : C. eriostachys presentó un cociente mayor en febrero y abril, y menor en las de septiembre y noviembre (Cuadro 3). En contraste, las muestras de C. eriostachys tuvieron un valor mayor en los cocientes $C: P(p=0.0001)$ y $N: P(p=0.0001)$ que $C$. elaeagnoides (Cuadro 3), al igual que las hojas vivas.

Cuadro 2. Media \pm un error estándar de algunos nutrientes y de sus cocientes en hojas vivas de dos especies de árboles remanentes en condiciones de pradera en Chamela, Jalisco en dos fechas diferentes. Letras distintas significan diferencias entre especies con la prueba de Tukey $(p=0.05$, $n=5)$.

\begin{tabular}{|l|c|c|c|c|}
\hline \multirow{2}{*}{} & \multicolumn{3}{|c|}{ Julio } & \multicolumn{2}{c|}{ Septiembre } \\
\cline { 2 - 5 } & C. elaeagnoides & C. eriostachys & C. elaeagnoides & C. eriostachys \\
\hline $\mathrm{C}\left(\mu \mathrm{g} \mathrm{g}^{-1}\right)$ & $416121(3620) \mathrm{b}$ & $450405(5295) \mathrm{a}$ & $434721(7885) \mathrm{b}$ & $462719(5671) \mathrm{a}$ \\
$\mathrm{N}\left(\mu \mathrm{g} \mathrm{g}^{-1}\right)$ & $36975(8116)$ & $41400(5127)$ & $29625(11813)$ & $33180(3904)$ \\
$\mathrm{P}\left(\mu \mathrm{g} \mathrm{g}^{-1}\right)$ & $5448(896) \mathrm{a}$ & $3267(526) \mathrm{b}$ & $4091(259) \mathrm{a}$ & $2605(259) \mathrm{b}$ \\
$\mathrm{C}: \mathrm{N}$ & $13(3.7)$ & $11(1.5)$ & $27(13)$ & $14(1.5)$ \\
$\mathrm{C}: \mathrm{P}$ & $83(14) \mathrm{b}$ & $149(18) \mathrm{a}$ & $109(11) \mathrm{b}$ & $177(8) \mathrm{a}$ \\
$\mathrm{N}: \mathrm{P}$ & $7(2.8) \mathrm{b}$ & $14(2.7) \mathrm{a}$ & $7(3.0) \mathrm{b}$ & $12(0.5) \mathrm{a}$ \\
Lignina (\%) & $10(2.0) \mathrm{a}$ & $3(0.1) \mathrm{b}$ & $8(1.8) \mathrm{a}$ & $5(1.1) \mathrm{b}$ \\
Celulosa (\%) & $29(1.8)$ & $27(1.2)$ & $35(1.2)$ & $31(2.9)$ \\
Polifenoles (\%) & $0.24(0.01) \mathrm{b}$ & $0.30(0.01) \mathrm{a}$ & $0.08(0.02) \mathrm{b}$ & $0.33(0.01) \mathrm{a}$ \\
Lig.:Cel. & $0.35(0.05) \mathrm{a}$ & $0.12(0.01) \mathrm{b}$ & $0.24(0.06) \mathrm{a}$ & $0.17(0.02) \mathrm{b}$ \\
Lig.:N & $353(99) \mathrm{a}$ & $82(11) \mathrm{b}$ & $409(102) \mathrm{a}$ & $107(31) \mathrm{b}$ \\
\hline
\end{tabular}

Las fracciones de C fueron diferentes entre las especies (Cuadro 3): C. elaeagnoides presentó mayor concentración de lignina y menor de polifenoles en las distintas fechas de colecta, al igual que las hojas vivas. En cambio, la celulosa fue afectada por la temporada: $C$. elaeagnoides tuvo menos celulosa en las muestras de febrero y abril, y más en las de septiembre y noviembre. Sin embargo, los cambios temporales de la celulosa, no se expresaron en el cociente lignina:celulosa, ya que $C$. elaeagnoides siempre resgistró un cociente mayor ( $p=0.001$; Cuadro 3 ).

En la Fig. 2 se muestra el patrón estacional de producción de hojarasca de cada una de las especies. El ANOVA indicó una interacción significativa $(p=0.05)$ : en las primeras colectas, que incluye el material depositado de noviembre de 1996 a abril de 1997, C. eriostachys elaboró mayor cantidad que $C$. elaeagnoides, pero en la segunda temporada (julio, septiembre y noviembre de 1997) tal relación fue inversa. Este patrón de producción de hojarasca determinó la magnitud de los flujos estacionales de nutrientes 
Cuadro 3. Media \pm un error estándar de algunos nutrientes y de sus cocientes en la hojarasca de dos especies de árboles remanentes en condiciones de pradera en Chamela, Jalisco. Distintas letras significan diferencias entre especies en cada fecha de colecta con la prueba de Tukey $(p=0.05, n=5)$. $\mathrm{El}$ * indica que los análisis químicos se hicieron en muestras compuestas.

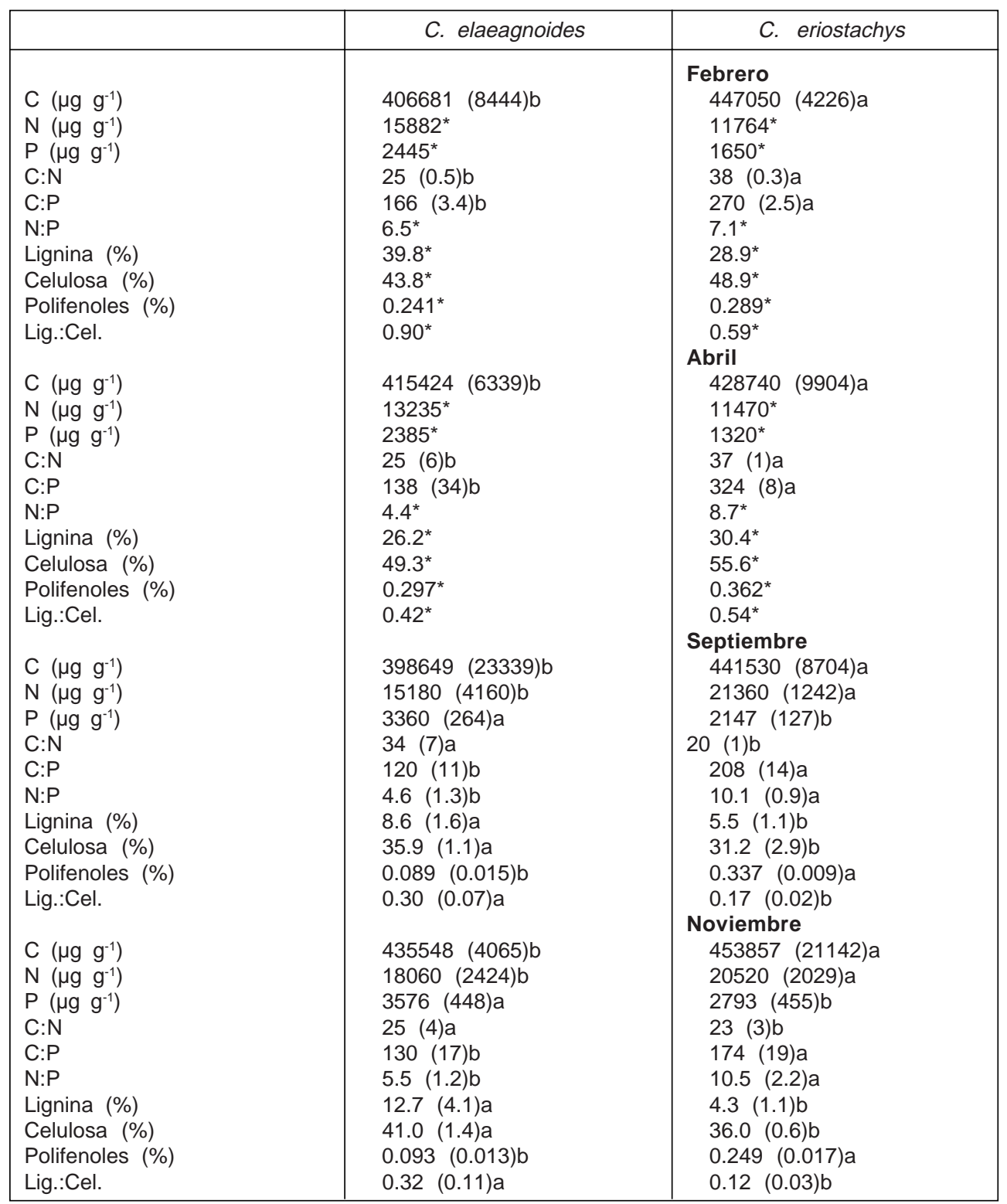




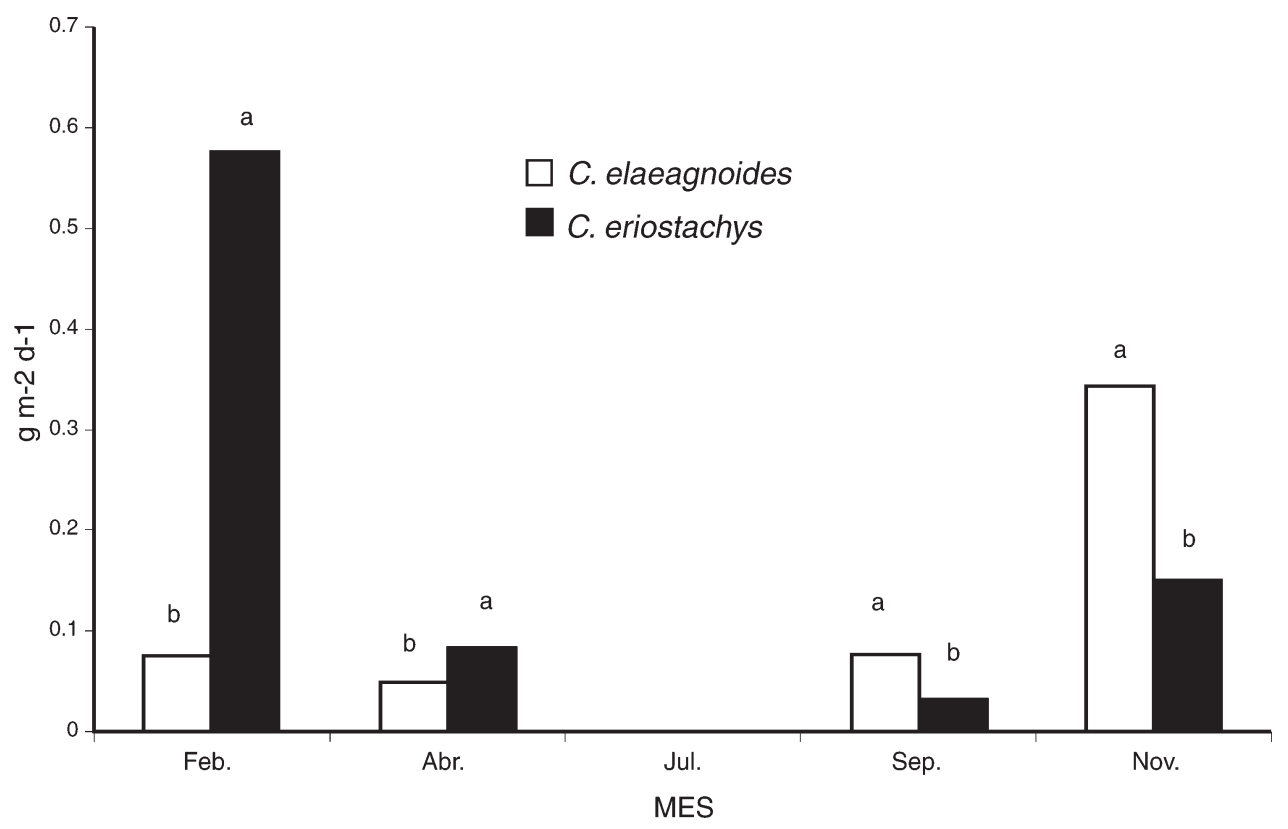

Fig. 2. Producción estacional de hojarasca $\left(\mathrm{g} \mathrm{m}^{-2} \mathrm{~d}^{-1}\right)$ de dos especies de árboles remanentes en condiciones de pradera en Chamela, Jalisco. Letras distintas significan diferencias entre especies con la prueba de Tukey $(\mathrm{p}=0.05 ; \mathrm{n}=5)$.

(Fig. 3). Considerando solamente los valores de la producción de hojarasca de junio a noviembre de 1997, no hubo diferencias entre las especies en la cantidad de materia orgánica, C, N y P que retornan al suelo. Sin embargo, C. elaeagnoides regresa mayor cantidad de los tres elementos, pues los valores registrados fueron $11.4 \pm 5.6$ y $5.3 \pm 0.9 \mathrm{~g}$ $\mathrm{C} \mathrm{m}^{-2}, 0.54 \pm 0.29$ y $0.24 \pm 0.05 \mathrm{~g} \mathrm{~N} \mathrm{~m}^{-2}$, y $0.08 \pm 0.03$ y $0.03 \pm 0.007 \mathrm{~g} \mathrm{P} \mathrm{m}^{-2}$ para esta especie y $C$. eriostachys, respectivamente. Tal patrón de los flujos puede explicarse en función de la producción de hojarasca más que de los cambios en la concentración de los nutrientes. Sin embargo, considerando los valores registrados entre noviembre de 96 y abril de 97, la relación entre las especies se invierte, mostrando mayores flujos $C$. eriostachys.

Aunque $C$. elaeagnoides presentó mayor flujo de $\mathrm{N}$ y $\mathrm{P}$ de junio a noviembre de 1997, cabe observar una diferencia en la eficiencia de uso de estos nutrientes entre las dos especies, pues $C$. elaeagnoides $(68 \pm 13)$ no la registró significativamente mayor en

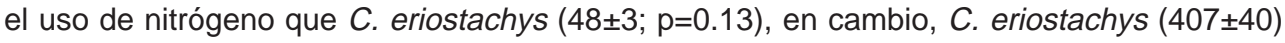
mostró una eficiencia significativamente mayor en el uso de fósforo que $C$. elaeagnoides $(296 \pm 28 ; p=0.02)$. 

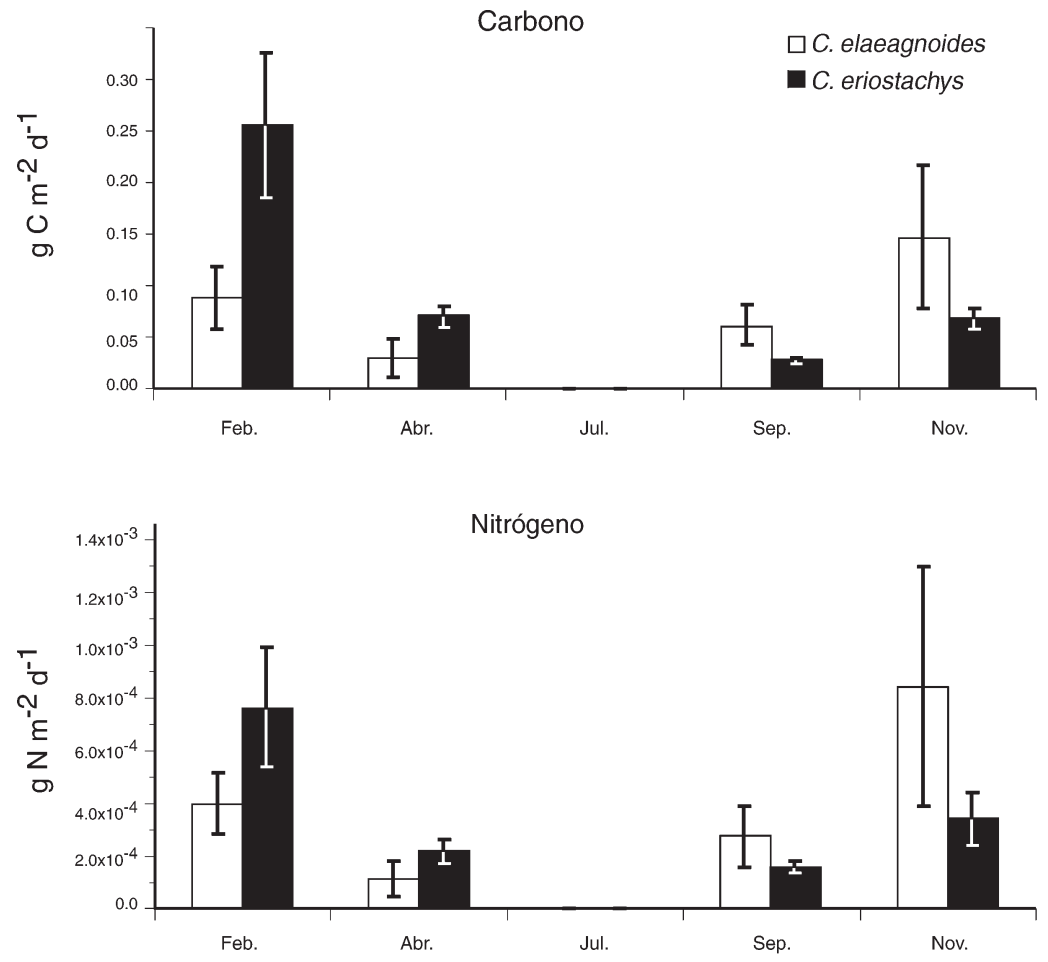

Fósforo

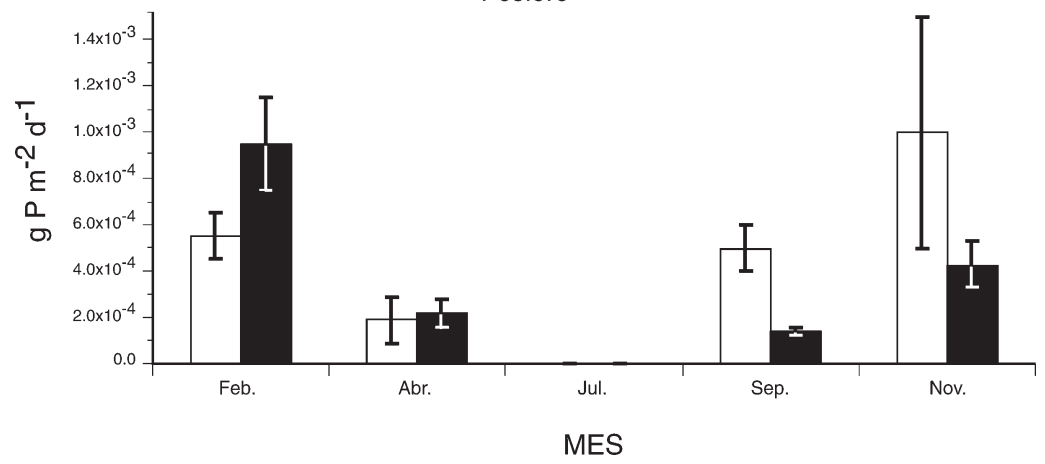

Fig. 3. Medias y error estándar $\left(\mathrm{g} \mathrm{m}^{-2} \mathrm{~d}^{-1}\right)$ de los flujos estacionales de carbono, nitrógeno y fósforo en la hojarasca de dos especies de árboles remanentes en condiciones de pradera en Chamela, Jalisco $(n=5)$. 
Galicia et al.: Flujos al Suelo de Dos Especies de Árboles en una Pradera Tropical Estacional

Dinámica del mantillo

En el Cuadro 4 se presentan las características químicas del mantillo en las diferentes fechas. No se registraron diferencias significativas en las concentraciones de $\mathrm{C}$ y $\mathrm{N}$ entre el mantillo de las dos especies ( $\mathrm{p}=0.2$ para ambos nutrientes). En cuanto al $\mathrm{P}, \mathrm{C}$. elaeagnoides presentó mayores valores que $C$. eriostachys en todas las fechas $(p=0.001)$. Los cocientes $C: N, C: P$ y N:P fueron mayores en el mantillo de $C$. eriostachys $(p=0.05, p=0.0001$ y $p=0.01$, respectivamente). El efecto de las especies no fue significativo en la concentración de lignina, pero sí en las de celulosa y polifenoles. $C$. eriostachys presentó una mayor concentración de polifenoles en todas las fechas $(p=0.0002)$ y la interacción del término especie por fecha afectó a la celulosa $(p=0.005)$ : en los meses de lluvia (julio y septiembre); su mayor concentración se resgistró en $C$. eriostachys. Como consecuencia, el cociente lignina:celulosa también fue afectado por la interacción, siendo mayor en $C$. eriostachys en los meses de abril y noviembre $(p=0.0003)$.

Entre julio y noviembre de 1997, C. elaeagnoides presentó mayor masa de mantillo en pie que $C$. eriostachys ( $p=0.05$, Fig. 4$)$, pero su tiempo de residencia fue menor $(0.020$ y 0.031 año $^{-1}$ para $C$. elaeagnoides y $C$. eriostachys, respectivamente). Los contenidos de $\mathrm{C}, \mathrm{N}$ y $\mathrm{P}$ siguen el mismo patrón que la cantidad del mantillo, con valores mayores debajo de $C$. elaeagnoides. Al igual que la masa del mantillo, los tiempos de residencia fueron bajos para N (0.017 y 0.021 año $^{-1}$ para $C$. elaeagnoides y $C$. eriostachys, respectivamente) y $\mathrm{P}\left(0.017\right.$ y $0.023 \mathrm{año}^{-1}$ para $C$. elaeagnoides y $C$. eriostachys, respectivamente).

\section{DISCUSIÓN}

Las concentraciones de los principales nutrientes en cada uno de los componentes de las dos especies de árboles sugieren la existencia de una dinámica diferencial. Por ejemplo $C$. elaeagnoides favorece una mayor circulación de $\mathrm{P}$ hacia el suelo que $C$. eriostachys, aunque el efecto de estos árboles no es muy claro sobre el reciclaje del $\mathrm{N}$. Tales cambios son consecuencia de la disponibilidad de nutrientes para la planta y/o de los ajustes fisiológicos que se realizan para mantener sus balances carbón-nutriente y nutriente-nutriente (Shaver y Melillo, 1984; Lajtha y Klein, 1988; Medina y Cuevas, 1989; Vitousek et al., 1995). La diferente dinámica de nutrientes se observó también en los valores de eficiencia de uso de $\mathrm{P}$, siendo ésta mayor en $C$. eriostachys. Lo último sugiere que debajo de los árboles de esta especie hay menor disponibilidad de $\mathrm{P}$, hecho que coincide con una menor concentración de $\mathrm{P}$ en su mantillo. Døckersmith et al. (1999) registraron una concentración de formas disponibles de $P$ en suelo asociado a $C$. eriostachys menor que la encontrada en el suelo asociado a Forchhammeria pallida en condiciones de selva sin perturbar en Chamela. Tal fenómeno podría ser explicado si $C$. eriostachys fuera una planta fijadora de $\mathrm{N}$, ya que las especies que presentan nódulos microbianos demandan más $P$ para el mantenimiento de la simbiosis (Binkley y Giardina, 1997). Hasta la fecha no se ha comprobado si $C$. eriostachys efectúa esta simbiosis; sin embargo, la mayor concentración de $\mathrm{N}$ en su follaje y una mayor capacidad buffer sobre los ácidos en el suelo asociado a ella (Nava-Mendoza et al., 2000) sugieren este hecho, mismo que está sujeto a una apropiada verificación. 
Cuadro 4. Media \pm un error estándar de algunos nutrientes y de sus cocientes en mantillo de dos especies de árboles remanentes en condiciones de pradera en Chamela, Jalisco. Distintas letras significan diferencias entre especies en cada fecha de colecta con la prueba de Tukey $(p=0.05, n=5)$. $\mathrm{El}$ * indica que los análisis químicos se hicieron en muestras compuestas.

\begin{tabular}{|c|c|c|}
\hline & C. elaeagnoides & C. eriostachys \\
\hline & & Abril \\
\hline$C\left(\mu g g^{-1}\right)$ & 366745 (20982) & 336676 (22570) \\
\hline$N\left(\mu g^{-1}\right)$ & $12941^{*}$ & $10000^{*}$ \\
\hline$P\left(\mu g^{-1}\right)$ & $2205^{*}$ & $945^{*}$ \\
\hline $\mathrm{C}: \mathrm{N}$ & $28(2) b$ & 33 (2)a \\
\hline$C: P$ & $160(9) b$ & $356(23) \mathrm{a}$ \\
\hline$N: P$ & $5.9^{*}$ & $10.5^{\star}$ \\
\hline Lignina (\%) & $41^{*}$ & $55^{*}$ \\
\hline Celulosa (\%) & $38^{*}$ & $29^{*}$ \\
\hline Polifenoles (\%) & $0.145^{\star}$ & $0.270^{\star}$ \\
\hline Lig.:Cel. & $1.09^{*}$ & $\begin{array}{c}1.88^{*} \\
\text { Julio }\end{array}$ \\
\hline$C\left(\mu g^{-1}\right)$ & 295680 (24092) & 283584 (15043) \\
\hline$N\left(\mu g^{-1}\right)$ & 20220 (3109) & $15600(3300)$ \\
\hline$P\left(\mu g^{-1}\right)$ & 2127 (306)a & $1350(114) \mathrm{b}$ \\
\hline$C: N$ & $15(2) b$ & 20 (3)a \\
\hline$C: P$ & $148(22) b$ & 206 (13)a \\
\hline$N: P$ & $10.3(2.1) \mathrm{a}$ & $11.0(1) \mathrm{a}$ \\
\hline Lignina (\%) & $3(0.9)$ & $8(1.4)$ \\
\hline Celulosa (\%) & $28(2.2) b$ & $32(1.3) \mathrm{a}$ \\
\hline Polifenoles (\%) & $0.030(0.001) \mathrm{b}$ & $0.071 \quad(0.001) a$ \\
\hline Lig.:Cel. & $0.13(0.03) b$ & $\begin{array}{l}0.28(0.05) a \\
\text { Septiembre }\end{array}$ \\
\hline$C\left(\mu g^{-1}\right)$ & 327668 (22749) & 326559 (7904) \\
\hline$N\left(\mu g^{-1}\right)$ & $14800(2401)$ & 11700 (1279) \\
\hline$P\left(\mu g^{-1}\right)$ & 3697 (369)a & $2480(371) b$ \\
\hline$C: N$ & $24(5) b$ & 30 (3)a \\
\hline$C: P$ & 91 (10)b & $147(25) a$ \\
\hline$N: P$ & $2.6(1) b$ & 5.2 (1)a \\
\hline Lignina (\%) & 11 (1.4) & $3(1.4)$ \\
\hline Celulosa (\%) & $35(0.7) \mathrm{b}$ & 48 (1.5)a \\
\hline Polifenoles (\%) & $0.058(0.001) \mathrm{b}$ & $0.123(0.004) a$ \\
\hline Lig.:Cel. & $0.33(0.03) \mathrm{a}$ & $\begin{array}{l}0.07(0.03) b \\
\text { Noviembre }\end{array}$ \\
\hline$C\left(\mu g g^{-1}\right)$ & 281083 (15662) & 254374 (17523) \\
\hline$N\left(\mu g^{-1}\right)$ & 14280 & 15600 \\
\hline$P\left(\mu g^{-1}\right)$ & 2553 (205)a & 1929 (239)b \\
\hline $\mathrm{C}: \mathrm{N}$ & 20 (2)a & 17 (2)a \\
\hline $\mathrm{C}: \mathrm{P}$ & $115(15) \mathrm{b}$ & 144 (27)a \\
\hline $\mathrm{N}: \mathrm{P}$ & $5.8(0.8) \mathrm{b}$ & $8.4(1.0) a$ \\
\hline Lignina (\%) & $20(6.2)$ & $14(4.9)$ \\
\hline Celulosa (\%) & $27(2.0)$ & $27(1.8)$ \\
\hline Polifenoles (\%) & $0.063(0.014) \mathrm{b}$ & $0.096(0.028) a$ \\
\hline Lig.:Cel. & $0.77(0.28) a$ & $0.49(0.13) b$ \\
\hline
\end{tabular}




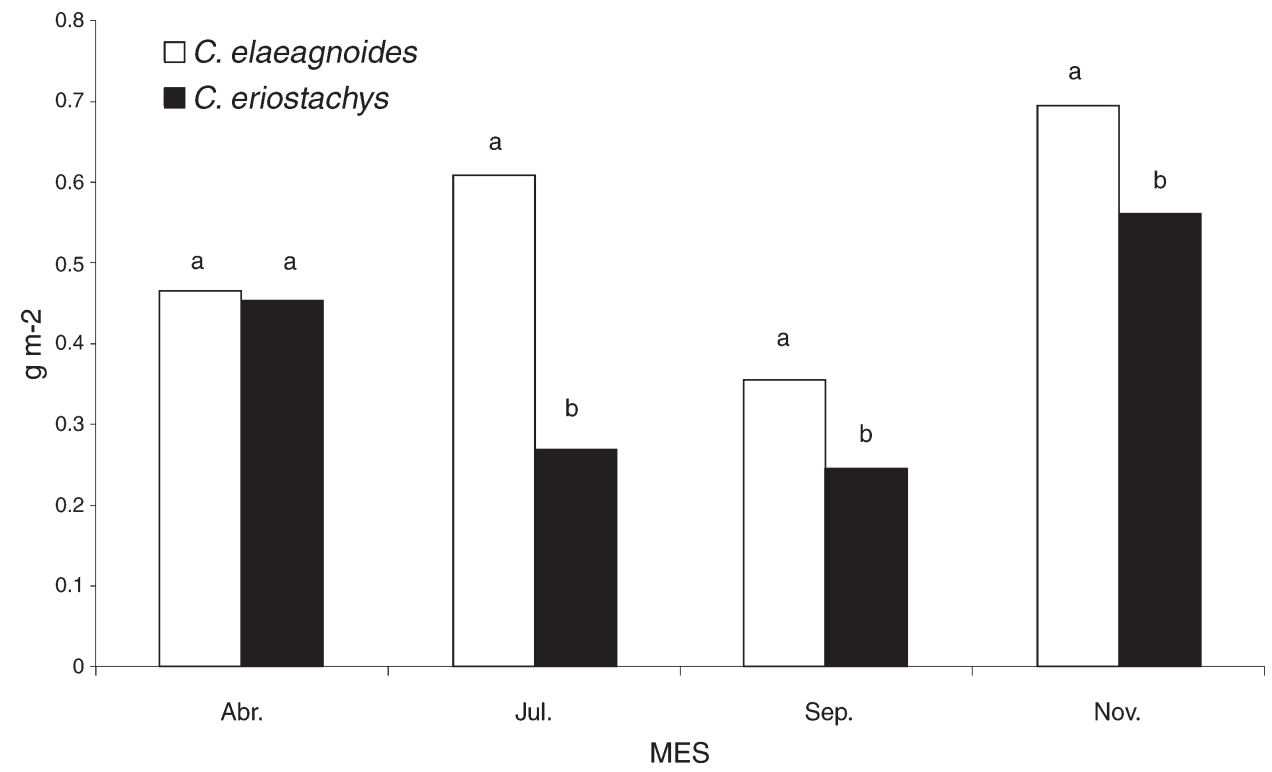

Fig. 4. La masa de mantillo $\left(\mathrm{g} \mathrm{m}^{-2}\right)$ de dos especies de árboles remanentes en condiciones de pradera en Chamela, Jalisco. Letras distintas significan diferencias entre especies con la prueba de Tukey $(p=0.05, n=5)$.

La diferencia en la magnitud de los flujos de nutrientes al suelo entre las dos especies depende de las divergencias de su producción anual de hojarasca: $C$. eriostachys registró un mayor flujo de nutrientes en la primera temporada, mientras que en la segunda lo efectuó $C$. elaeagnoides, lo que sugiere que la cantidad de nutrientes que retornan al suelo varía entre años. A pesar de que $C$. eriostachys presentó menor biomasa de mantillo en pie que C. elaeagnoides (Fig. 4), no hubo diferencias significativas en el tiempo de residencia de la materia orgánica, el cual determina el balance entre las entradas por hojarasca y las salidas por la descomposición en un ciclo temporal (Martínez-Yrízar y Sarukhán, 1993). Los valores de tiempo de residencia de ambas especies $(0.020$ y 0.031 año-1 para $C$. elaeagnoides y $C$. eriostachys, respectivamente) son bajos en comparación con lo señalado para la selva en Chamela (2.1 año-1 en Martínez-Yrízar y Sarukhán, 1993; 1.37 en Jaramillo y Sanford, 1995). Tal hecho sugiere que no existe acumulación de la materia orgánica de un año a otro, debido a su rápida descomposición y remoción por erosión hídrica. Así, Martínez-Yrízar (1984) registró altas tasas de descomposición del mantillo de C. eriostachys, ya que $50 \%$ de la masa se perdió en 38 días, una vez iniciada la temporada de lluvias. Aunado a lo anterior, la erosión hídrica debe tener un efecto significativo en la desaparición del mantillo, ya que es muy importante en las condiciones abiertas de la pradera (Maass 
et al., 1988; García-Oliva et al., 1995b). De igual manera, los tiempos de residencia de $\mathrm{N}$ y $\mathrm{P}$ son muy cortos en ambas especies, siendo menores en $\mathrm{C}$. elaeagnoides. Cuando los tiempos de residencia de los nutrientes son menores que los de la materia orgánica, entonces se sugiere que la toma de nutrientes por las plantas domina sobre la inmovilización microbiana (Vogt et al., 1986), como es el caso particular del mantillo asociado a ambas especies estudiadas.

Sin embargo, la calidad del material asociado a la concentración de los nutrientes en cada una de las especies no es afectada por la variación anual, ya que $C$. elaeagnoides siempre presentó mayor concentración de $\mathrm{P}$ en todas sus fracciones foliares. La concentración de los nutrientes influye también en la dinámica de las poblaciones microbianas (Melillo et al., 1986; Parton et al., 1987); así, se encontró dos veces más bacterias y actinomicetos en el mantillo asociado a los árboles de $C$. eriostachys que en el ligado con los de C. elaeagnoides (E. Rivas, com. pers.), pero el suelo debajo de las plantas de $C$. eriostachys presentó la mitad del valor de $C$ encontrado en la biomasa microbiana que aquel debajo de la influencia de $C$. elaeagnoides (Galicia, 2001). Asimismo, las diferentes especies definen de una manera distinta el tipo de nutrientes que forman parte del complejo de intercambio catiónico del suelo, ya que $70 \%$ de las superficies de tal intercambio estuvieron ocupadas por cationes alcalinos en el suelo asociado a C. elaeagnoides, mientras que sólo $41 \%$ de tales superficies correspondió al suelo de C. eriostachys (Nava-Mendoza et al., 2000). Todo lo anterior tiene gran influencia en la dinámica de los nutrientes del suelo.

En el mantillo, la concentración de polifenoles también fue distinta para las dos especies, pero los valores más altos que encontramos $(0.27 \%$, Cuadro 4$)$ fueron inferiores a los que reducen la tasa de descomposición (mayores de 3\%; según Palm y Sánchez, 1990). Lo anterior sugiere que la concentración de los polifenoles en ambas especies estudiadas no afecta la tasa de descomposición, como se ha encontrado para otros árboles tropicales de la familia Leguminosae (Palm y Sánchez, 1990).

Aunque tomando en cuenta las diferencias entre ambas especies, el suelo asociado a ellas mantuvo mayor contenido de nutrientes y dinámica microbiana que el suelo en la pradera, lo que sugiere que cada uno de sus individuos favorece el mantenimiento del ciertos mecanismos de protección de nutrientes en su área de influencia, los cuales se pierden en la pradera (García-Oliva et al., 1999a; NavaMendoza et al., 2000; Galicia, 2001). Lo anterior resalta la importancia de los árboles remanentes en favorecer una mayor cantidad de procesos ecológicos, así como una mayor diversidad de plantas debajo de su copa, como ya ha sido señalado para ecosistemas tropicales húmedos (McDonell, 1986; Campbell et al., 1990, Guevara et al., 1992; Otero-Arnaiz et al., 1999).

En conclusión, el flujo de nutrientes en las dos especies fue variable entre años, dependiendo de su productividad en cada año en particular. Sin embargo, los efectos de esta variación anual probablemente no son significativos en virtud de los cortos tiempos de residencia del mantillo en el suelo. Por otro lado, la concentración de nutrientes fue diferente en las especies en ambas temporadas, pues $C$. elaeagnoides mantuvo mayores valores de $P$. 
Galicia et al.: Flujos al Suelo de Dos Especies de Árboles en una Pradera Tropical Estacional

\section{AGRADECIMIENTOS}

El presente trabajo forma parte de la tesis de licenciatura de Ramón Murillo y de la tesis de doctorado de Leopoldo Galicia. Los autores agradecemos al editor y a dos revisores anónimos, a Angelina Martínez-Yrízar y Sonia Álvarez-Santiago por sus comentarios al manuscrito, a María Paz Tapia y Salvador Araiza por su ayuda en el trabajo de campo, a Georgina García-Méndez, Maribel Nava-Mendoza y Adriana Hernández por su apoyo en el laboratorio, a Heberto Ferreira por su asistencia en el procesamiento de los datos. También damos las gracias al personal de la Estación de Biología Chamela por todo el soporte logístico durante el trabajo de campo y a Ramiro Peña por permitirnos usar sus parcelas. El financiamiento de este proyecto corrió a cargo de DGAPA-UNAM (IN-200797).

\section{LITERATURA CITADA}

Anderson, J. M. y J. S. I. Ingram. 1993. Tropical soil biology and fertility. A handbook of methods. $2 \mathrm{a}$ ed. CAB International Oxford. $221 \mathrm{pp}$.

Anónimo. 1977. Technicon publication methods No. 329-74W/B. Individual /simultaneous determinations of nitrogen and/or phosphorus in BD acid digest. Technicon Industrial System, Nueva York. $150 \mathrm{pp}$.

Belsky, A. J. 1994. Influences of trees on savanna productivity: test of shade, nutrients, and treegrass competition. Ecology 75: 922-932.

Binkley, D. y D. Valentine. 1991. Fifty-year biogeochemical effects of green ash, white pine, and Norway spruce in a replicated experiment. For. Ecol. Manage. 40: 13-25.

Binkley, D., P. Sollins, R. Bell, D. Sachs y D. Myrold. 1992. Biogeochemistry of adjacent conifer and alder-conifer stands. Ecology 73: 2022-2033.

Binkley, D. y C. Giardina. 1997. Nitrogen fixation in tropical forest plantation. In: Nambiar, E. K. y A. G. Brown (eds.). Management of soil, nutrients and water in tropical plantation forest. Australian Center for International Agricultural Research, Camberra. pp. 297-338.

Bullock, S. H. 1986. Climate of Chamela, Jalisco and trends in the south coastal region of Mexico. Arch. Meteorol. Geophys. Bioclimatol. 36: 297-316.

Bullock, S. H. y A. Solís-Magallanes. 1990. Phenology of canopy trees of a tropical deciduous forest in Mexico. Biotropica 21: 22-35.

Burke, I. C., W. A. Reiners y D. S. Schimel. 1989. Organic matter turnover in a sagebrush steppe landscape. Biogeochemistry 7: 11-31.

Campbell, B. M., T. Lyam y J. C. Hatton. 1990. Small-scale patterning in the recruitment of forest species during succession in tropical dry forest, Mozambique. Vegetatio 87: 51-57.

Campo, J., J. M. Maass y L. de Pablo. 2001. Intemperismo en un bosque tropical seco de México. Agrociencia 35: 245-254.

Døckersmith, I. C., C. P. Giardina y R. L. Sanford. 1999. Persistence of tree related patterns in soil nutrients following slash-and-burn disturbance in the tropics. Plant Soil 209: 137-156.

Galicia, L. 2001. Efecto de dos especies de árboles remanentes en la entrada de $\mathrm{C}$ y $\mathrm{N}$ al suelo en una pradera de un ecosistema tropical estacional. Tesis de doctorado, Instituto de Ecología, Universidad Nacional Autónoma de México. México, D.F. 128 pp.

García-Miragaya, J., S. Flores y N. Chacón. 1994. Soil chemical properties under individual evergreen and deciduous trees in a protected Venezuelan savanna. Acta Oecologica 15: 477-484.

García-Montiel, D. y D. Binkley. 1998. Effect of Eucalyptus saligna and Albizia falcataria on soil processes and nitrogen supply in Hawai. Oecologia 113: 547-556. 
García-Moya E. y C. M. McKell. 1970. Contribution of shrubs to the nitrogen economy of a desert-wash plant community. Ecology 51: 81-88.

García-Oliva, F., E. Ezcurra y L. Galicia. 1991. Pattern of rainfall distribution in the central Pacific coast of Mexico. Geografiska Annaler 73A: 179-186.

García-Oliva, F., I. Casar, P. Morales y J. M. Maass. 1994. Forest-to-pasture conversion influences on soil organic carbon dynamics in a tropical deciduous forest. Oecologia 99: 392-396.

García-Oliva, F., J. M. Maass y L. Galicia. 1995a. Rainstorm analysis and rainfall erosivity of a seasonal tropical region with a strong cyclonic influence on the Pacific coast of Mexico. J. Appl. Meteor. 34: 2491-2498.

García-Oliva, F., R. Martínez y J. M. Maass. 1995b. Long-term net soil erosion as determined by ${ }^{137}$ Cs redistribution in an undisturbed and perturbed tropical deciduous forest ecosystem. Geoderma 68:135-147.

García-Oliva, F. y J. M. Maass. 1998. Efecto de la transformación de la selva a pradera sobre la dinámica de los nutrientes en un ecosistema tropical estacional en México. Bol. Soc. Bot. México 62: $39-48$

García-Oliva, F., R. L. Sanford Jr. y E. Kelly. 1999a. Effects of slash-and-burn management on soil aggregate organic $\mathrm{C}$ and $\mathrm{N}$ in a tropical deciduous forest. Geoderma 88: 1-12.

García-Oliva, F., R. L. Sanford Jr. y E. Kelly. 1999b. Effect of burning of tropical deciduous forest soil in Mexico on the microbial degradation of organic matter. Plant Soil 206: 29-36.

Giardina, C., R. L. Sanford Jr. y I. C. Døckersmith. 2000. Changes in soil phosphorus and nitrogen during slash-and-burn clearing of a dry tropical forest. Soil Sci. Soc. Am. J. 64: 399-405.

González-Flores, P.C. 1992. El manejo del fuego en el sistema de roza, tumba y quema en la selva baja caducifolia de Chamela, Jalisco. Tesis de Licenciatura, Facultad de Ciencias, Universidad Nacional Autónoma de México. México, D.F. 99 pp.

Gutiérrez-Alcalá, A. R. 1993. La ganadería extensiva en el trópico seco mexicano: causas, consecuencias y manifestaciones en su medio social. Tesis de Licenciatura, Facultad de Filosofía y Letras, Universidad Nacional Autónoma de México. México, D.F. 78 pp.

Guevara, S., J. Meave, P. Moreno-Casasola y J. Laborde. 1992. Floristic composition and structure of vegetation under isolates trees in neotropical pastures. J. Veg. Sci. 3: 655-664.

Hobbie, S. E. 1992. Effects of plant species on nutrient cycling. Tree 7: 336-339.

Jaramillo, V. J. y R. L. Sanford 1995. Nutrient cycling in tropical deciduous forests. In: Bullock, S. H., H. A. Mooney y E. Medina (eds.). Seasonally dry tropical forests. Cambridge University Press. Cambridge. pp. 346-361.

Kellman, M. 1979. Soil enrichment by neotropical savanna trees. J. Ecol. 67: 565-577.

Koch, A. S. y E. Matzner. 1993. Heterogeneity of soil and soil solution chemistry under Norway spruce (Picea abies Karst.) and European beech (Fagus silvatica L.) as influenced by distance from the stem basis. Plant Soil 151: 227-237.

Lajtha, K. y M. Klein. 1988. The effect of varying nitrogen and phosphorus availability on nutrient use of Larrea tridentata, a desert evergreen shrub. Oecologia 75: 348-353.

Lott, E. J. 1993. Annotated checklist of the vascular flora of the Chamela bay region, Jalisco, Mexico. Occasional Paper of the California Academy of Sciences 148: 1-60.

Maass, J. M., C. Jordan y J. Sarukhán. 1988. Soil erosion and nutrient losses in a seasonal tropical agroecosystems under various management techniques. J. Appl. Ecol. 25: 595-607.

Martínez-Yrízar, A. 1984. Procesos de producción y descomposición de hojarasca en selvas estacionales. Tesis de Maestría, Facultad de Ciencias, Universidad Nacional Autónoma de México. México, D.F. 58 pp.

Martínez-Yrízar, A. y J. Sarukhán. 1990. Literfall patterns in a tropical deciduous forest in Mexico over a five-year period. J. Trop. Ecol. 6: 433-444.

Martínez-Yrízar, A. y J. Sarukhán. 1993. Cambios estacionales del mantillo en el suelo de un bosque tropical caducifolio y uno subcaducifolio en Chamela, Jalisco, México. Acta Bot. Mex. 21: $1-16$. 
Martínez-Yrízar, A., J. M. Maass, L. A. Pérez-Jiménez y J. Sarukhán. 1996. Net primary productivity of a tropical deciduous forest ecosystem in western Mexico. J. Trop. Ecol. 12: 169-175.

McDonell, M. J. 1986. Old field vegetation height an the dispersal pattern of bird-disseminated woody plants. Bull. Torrey Bot. Club 113: 6-11.

Medina, E. y E. Cuevas. 1989. IV. Mineral nutrition: humid tropical forest. Prog. Bot. 55: 115-129.

Melillo, J. M., J. D. Aber y J. F. Muratore. 1982. Nitrogen and lignin control of harwood leaf litter decomposition dynamics. Ecology 63: 621-626.

Miles, J. 1985.The pedogenic effects of different species and vegetation types an the implications of succession. J. Soil Sci. 36: 571-584.

Miranda, F. y E. Hernández X. 1963. Los tipos de vegetación en México y su clasificación. Bol. Soc. Bot. México 28: 29-179.

Montgomery, D. C. 1984. Design and analysis of experiments. 2a ed. John Wiley \& Sons. Nueva York. $538 \mathrm{pp}$.

Nava-Mendoza, M., L. Galicia y F. García-Oliva. 2000. Efecto de dos especies de árboles remanentes y de un pasto en la capacidad amortiguadora del $\mathrm{pH}$ del suelo en un ecosistema tropical estacional. Bol. Soc. Bot. México 67: 17-24.

Newbold, P. J. 1967. Methods for estimating the primary production of forest. IBP Handbook No. 2. Blackwell Scientific Publication. Oxford. $62 \mathrm{pp}$.

Otero-Arnaiz, A., S. Castillo, J. Meave y G. Ibarra-Manríquez. 1999. Isolated pastures trees and vegetation under their canopies in the Chiapas Coastal Plain, México. Biotropica 31: 243-254.

Palm, C. A. y P. A. Sánchez. 1990. Decomposition and nutrient release patterns of the leaves of three tropical legumes. Biotropica 22: 330-338.

Parton, W. J., D. S. Schimel, C. V. Cole y D. S. Ojima. 1987. Analysis of factors controlling soil organic matter levels in Great Plains grassland. Soil Sci. Soc. Am. J. 51: 1173-1179.

Pastor, J., J. D. Aber, C. A. McClaugherty y J. M. Melillo. 1984. Aboveground production and $\mathrm{N}$ and $\mathrm{P}$ cycling along a nitrogen mineralization gradient on Blackhawk Island, Wisconsin. Ecology 65: 256-268.

Rodríguez, R. 1999. Cartografía morfométrica jerárquica a tres escalas del área microbloque "El Colorado" Chamela, Jalisco. Tesis de Licenciatura, Facultad de Filosofía y Letras, Universidad Nacional Autónoma de México. México, D.F. 136 pp.

Shaver, G. R. y J. M. Melillo. 1984. Nutrient budgets of marsh plants: efficiency concept and relation to availability. Ecology 65: 1491-1510.

Solís, E. 1993. Características fisicoquímicas de un suelo en un ecosistema tropical estacional. Tesis de Licenciatura, Facultad de Ciencias, Universidad Nacional Autónoma de México. México, D.F. $91 \mathrm{pp}$. 